\title{
Acoustofluidics 17: Theory and applications of surface acoustic wave devices for particle manipulation
}

\author{
Michael Gedge and Martyn Hill
}

${ }_{5}$ Engineering Sciences, University of Southampton, Southampton SO17 1BJ.

E-mail: m.hill@ soton.ac.uk. Tel: +44 2380593075

\begin{abstract}
In this paper, number 17 of the thematic tutorial series "Acoustofluidics - exploiting ultrasonic standing waves, forces and acoustic streaming in microfluidic systems for cell and particle manipulation" - we present the theory of surface acoustic waves (SAWs) and some related 10 microfluidic applications. The equations describing SAWs are derived for a solid-vacuum interface before generalisations are made about solid-solid and solid-fluid interfaces. Techniques for SAW generation are discussed before an overview of applications is presented.
\end{abstract}

\section{A. Introduction}

The theory of surface waves was first 15 discussed by Lord Rayleigh in the late $19^{\text {th }}$ century $^{1}$. It was shown that their effect decreased rapidly with depth and that their velocity of propagation is smaller than that of body waves ${ }^{2}$. As 20 these waves spread only in two dimensions their amplitude decays more slowly with distance than other types of elastic waves. At the time they were recognised as important in seismology

25 but they have since found use at much smaller scales, such as ultrasonic surface testing and in microfluidics. They also have significant use in electronic filters and sensors ${ }^{3}$. It will be shown that 30 Rayleigh waves are a combination of longitudinal motion and transverse motion confined to the surface of an elastic medium, penetrating only to about one wavelength in depth. Surface waves 35 on a solid are similar to surface waves on a liquid, in that particle motion is elliptical; however there are differences in direction and restoring forces. In solids it is elastic forces while in liquids it is 40 gravity and surface tension that supply that restoring force ${ }^{2}$.

Lamb waves will not be considered here, but it can be shown that a Lamb wave transforms into a Rayleigh wave as the 45 thickness of the substrate increases ${ }^{4}$. Key equations describing leaky Rayleigh waves and associated attenuation will be presented and discussed. They will show that shear based attenuation and Solid-

${ }_{50}$ Gas interface losses can largely be ignored.

Other Rayleigh-like waves such as Scholte waves, interface waves and Stoneley waves will be presented and 55 their use in particle manipulation techniques will be described. The paper then discusses the effects of piezoelectric substrates and anisotropy before detailing SAW generation methods. An overview 60 of current particle manipulation devices will then be given, covering planar microfluidics, SAW induced flow and radiation force based manipulation. A short section will describe Stoneley wave 65 based manipulation and the associated benefits.

The choice of using surface acoustic wave (SAW) devices as opposed to the alternative bulk acoustic wave (BAW) 70 devices for ultrasonic particle manipulation depends on a number of factors. SAW devices have the ability to work at very high frequencies, giving the potential for tighter control of particles. 75 Many SAW devices use standing surface acoustic waves to create the potential landscapes required for manipulation so they do not rely on highly resonant chambers to provide the energy gradients. ${ }_{80}$ This allows the use of common but acoustically damped microfluidic materials such as PDMS. As the energy within SAWs is inherently confined to the substrate surface prior to launching into 85 the chamber, it is likely that they dissipate less energy in the microfluidic structure as a whole than is the case with BAW devices. While SAW fabrication lends itself to microfluidic integration there is 90 an additional cost and complexity involved than in the construction of BAW devices. Further, the resonant nature and lower frequencies of most BAW devices makes them more suitable for handling 95 larger volumes of fluid.

\section{B. Rayleigh Waves}

In an unbounded isotropic solid only two types of elastic wave can propagate, as shown in part 3 of this tutorial series ${ }^{5}$. 100 However, surface and interface waves exist at free boundaries and interfaces respectively, allowing Rayleigh waves to be used to manipulate particles and fluids when used in a microfluidic device.

105 The equations of motion used to describe longitudinal and transverse waves in an isotropic unbounded medium will be manipulated to generate equations detailing the displacements of a surface 110 wave propagating along a half space. Rotation phenomena and dilation phenomena will be decoupled and the Rayleigh wave number defined in terms of longitudinal and transverse wave 115 numbers.

The equation of motion for an unbounded 
isotropic elastic solid in which body forces are absent can be written $\mathrm{as}^{2}$ :

$$
\rho \frac{\partial^{2} u}{\partial t^{2}}=(\lambda+\mu) \frac{\partial \Delta}{\partial x}+\mu \nabla^{2} u,
$$

where $u$ defines displacement in the $x$ 5 direction. Similarly $v$ and $w$ are displacements in the $y$ and $z$ directions respectively. $\nabla^{2}$ is the Laplace operator whilst $\rho, \lambda$ and $\mu$ define the density, Lamé's constant and shear modulus of the 10 medium. $\Delta$, see Eq. (3), defines the volume strain of the medium. As an isotropic, unbounded solid is being considered, similar expressions can be written for $v$ and $w$. The solutions to 15 these equations define the longitudinal wave speed $\left(c_{\mathrm{L}}\right)$ and transverse, or shear, wave speed $\left(c_{\mathrm{T}}\right)$ in the $x$ direction:

$$
C_{\mathrm{L}}^{2}=\frac{\lambda+2 \mu}{\rho}, \quad C_{\mathrm{T}}^{2}=\frac{\mu}{\rho} .
$$

Following the approach of Kolsky ${ }^{2}$ the 20 same equations will be used to generate the equations of motion for a surface wave propagating in the $x$ direction on a half space. Take $z$ to be positive towards the interior of the half space and make 25 the $x y$ plane the free boundary of that half space. The wave will be polarized in the $x z$ plane, so there are no displacements in the $y$ direction, see Figure 1. This leads to the following 30 equations describing the volume strain, or dilation $\Delta$ of the medium,

$$
\Delta=\frac{\partial u}{\partial x}+\frac{\partial w}{\partial z}
$$

and the rotation $\Omega_{\mathrm{y}}$ in the $x z$ plane

$$
\Omega_{\mathrm{y}}=\frac{\partial u}{\partial z}-\frac{\partial w}{\partial x} \text {. }
$$

35 From here it is possible to define two scalar functions $\boldsymbol{\varphi}$ and $\boldsymbol{\psi}$ so that dilation and rotation effects can be decoupled

$$
\begin{aligned}
& u=\frac{\partial \varphi}{\partial x}+\frac{\partial \psi}{\partial z}, \\
& w=\frac{\partial \varphi}{\partial z}-\frac{\partial \psi}{\partial x} .
\end{aligned}
$$

This allows for dilation and rotation to be defined as $\boldsymbol{\nabla}^{2} \varphi$ and $\boldsymbol{\nabla}^{2} \psi$ respectively. By substituting Eqs.(5a) and (5b) into Eq.(1) it is seen that the equations of motion for ${ }_{45} \boldsymbol{u}$ and $\boldsymbol{w}$ are satisfied given that

$$
\begin{gathered}
\frac{\partial^{2} \varphi}{\partial t^{2}}=\frac{\lambda+2 \mu}{\rho} \nabla^{2} \varphi=C_{\mathrm{L}}^{2} \nabla^{2} \varphi, \\
\frac{\partial^{2} \psi}{\partial t^{2}}=\frac{\mu}{\rho} \nabla^{2} \psi=C_{\mathrm{T}}^{2} \nabla^{2} \psi .
\end{gathered}
$$

Now consider a sinusoidal wave of 50 angular frequency $\omega$ and wave number $k$ propagating in the $x$ direction and look for solutions of $\varphi$ and $\psi$ of the form

$$
\begin{aligned}
& \varphi=F(z) e^{\mathrm{i}(\omega t-k x)}, \\
& \psi=G(z) e^{\mathrm{i}(\omega t-k x)} .
\end{aligned}
$$

These trial solutions can be substituted into equations (6a) and (6b) which after re-arranging give the following

$$
\frac{\partial^{2}}{\partial z^{2}} F(z)-\left(k^{2}-k_{\mathrm{L}}^{2}\right) F(z)=0
$$

60

$$
\frac{\partial^{2}}{\partial z^{2}} G(z)-\left(k^{2}-k_{\mathrm{T}^{2}}\right) G(z)=0,
$$

where $k_{\mathrm{L}}$ and $k_{\mathrm{T}}$ are the longitudinal and transverse wave numbers respectively.

The longitudinal and transverse wave 65 numbers can be expressed in the following format:

$$
k_{\mathrm{L}}=\frac{\omega}{C_{\mathrm{L}}}, \quad k_{\mathrm{T}}=\frac{\omega}{C_{\mathrm{T}}} .
$$

Hence:$$
70
$$

$$
\frac{k_{\mathrm{L}}^{2}}{k_{\mathrm{T}}^{2}}=\frac{\mu}{\lambda+2 \mu}=\frac{1-2 v}{2-2 v}=\alpha_{1}{ }^{2},
$$

where in the third expression we have introduced the Poisson's ratio given by

$$
v=\frac{\lambda}{2(\lambda+\mu)} \text {. }
$$

Hence the longitudinal wave number can be expressed as the product of the transverse wave number $\left(k_{\mathrm{T}}\right)$ and a function of Poisson's ratio $\left(\alpha_{1}\right)$

80

$$
k_{\mathrm{L}}=\alpha_{1} k_{\mathrm{T}} .
$$

It is known that $k_{\mathrm{L}}^{2}<k_{\mathrm{T}}^{2}$ and it is assumed that $k_{\mathrm{T}}^{2}<k^{2}$ (this will be confirmed later).

${ }_{85}$ To determine the dispersion relation of $\omega$ in terms of $k$, we seek solutions of Eqs. (8a) and (8b) of the form

$$
F(z)=A \mathrm{e}^{\left(-\gamma_{\llcorner} z\right)}+A^{\prime} \mathrm{e}^{\left(\gamma_{\llcorner} z\right)},
$$

$$
G(z)=B \mathrm{e}^{\left(-\gamma_{\top} Z\right)}+B^{\prime} \mathrm{e}^{\left(\gamma_{\top} z\right)},
$$

where $\gamma_{\mathrm{L}}^{2}=k^{2}-k_{\mathrm{L}}^{2}$ for the longitudinalRayleigh combination and $\gamma_{\mathrm{L}}^{2}=k^{2}-$ $k_{\mathrm{T}}^{2}$ for the transverse-Rayleigh combination, and where $A, B, A^{\prime}$ and $B^{\prime}$ 95 are constants. The $A^{\prime}$ and $B^{\prime}$ components of these equations are physically unrealistic as they would lead to an increase in displacement amplitude with increasing depth, $z$. This leads to the 100 following equations describing $\varphi$ and $\psi$

$$
\begin{aligned}
& \varphi=A \mathrm{e}^{\left(-\gamma_{\mathrm{L} Z}\right)} \mathrm{e}^{\mathrm{i}(\omega t-k x)}, \\
& \psi=B \mathrm{e}^{\left(-\gamma_{\top} Z\right)} \mathrm{e}^{\mathrm{i}(\omega t-k x)} .
\end{aligned}
$$

105 Boundary conditions can now be applied that allow the elimination of $A$ and $B$, which ultimately leads to the determination of the displacements in the $x$ and $z$ directions. Plane stress and shear 110 stress will be equal to zero at the free surface, i.e. when $z$ is equal to zero. For plane stress:

$$
\sigma_{\mathrm{zz}}=\lambda \Delta+2 \mu \frac{\partial w}{\partial z}
$$

which can be expressed in terms of $\varphi$ 115 and $\psi$ : 
$\sigma_{\mathrm{zz}}=(\lambda+2 \mu) \frac{\partial^{2} \varphi}{\partial z^{2}}+\lambda \frac{\partial^{2} \varphi}{\partial x^{2}}-2 \mu \frac{\partial^{2} \psi}{\partial x \partial z}$.

Inserting the decaying components of equations (14a) and (14b) yields for $z$ equals zero:

$A\left[(\lambda+2 \mu) \gamma_{\mathrm{L}}+\lambda k^{2}\right]-2 \mu B \mathrm{i} \gamma_{\mathrm{T}} k=0$.

A similar approach is then taken for shear stress in the half space

$$
\sigma_{\mathrm{zx}}=\mu\left(\frac{\partial u}{\partial z}+\frac{\partial w}{\partial x}\right)
$$

10 which again can be expressed in terms of $\varphi$ and $\psi$

$$
\sigma_{\mathrm{zx}}=\mu\left(2 \frac{\partial^{2} \varphi}{\partial x \partial z}-\frac{\partial^{2} \psi}{\partial x^{2}}+\frac{\partial^{2} \psi}{\partial z^{2}}\right) .
$$

Inserting the decaying components of 15 equations (14a) and (14b) yields for $Z$ equals zero

$$
2 \mathrm{i} \gamma_{\mathrm{L}} k A+\left(\gamma_{\mathrm{T}^{2}}+k^{2}\right) B=0 .
$$

20 Equations (17) and (20) can be combined to eliminate $A$ and $B$, yielding

$$
4 \mu \gamma_{\mathrm{L}} \gamma_{\mathrm{T}} k^{2}=\left[(\lambda+2 \mu) \gamma_{\mathrm{L}}^{2}-\lambda k^{2}\right]\left(\gamma_{\mathrm{T}}^{2}+k^{2}\right) .
$$

${ }_{25}$ Squaring both sides of the equation and inserting expressions for $\gamma_{\mathrm{L}}$ and $\gamma_{\mathrm{T}}$ yields:

$$
\begin{gathered}
16\left(1-\frac{k_{\mathrm{L}}^{2}}{k^{2}}\right)\left(1-\frac{k_{\mathrm{T}}^{2}}{k^{2}}\right)= \\
{\left[2-\frac{(\lambda+2 \mu)}{\mu} \frac{k_{\mathrm{L}}^{2}}{k^{2}}\right]^{2}\left(2-\frac{k_{\mathrm{T}}^{2}}{k^{2}}\right)^{2}}
\end{gathered}
$$

Using Eqs.(10) and (12) this expression 30 can be written as

$$
16\left(1-\frac{\alpha_{1}^{2} k_{\mathrm{T}}^{2}}{k^{2}}\right)\left(1-\frac{k_{\mathrm{T}}^{2}}{k^{2}}\right)=\left(2-\frac{k_{\mathrm{T}}^{2}}{k^{2}}\right)^{4} .
$$

Expanding and replacing $k_{\mathrm{T}} / k$ with $s$ yields

35

$$
\begin{gathered}
s^{6}-8 s^{4}+\left(24-16 \alpha_{1}^{2}\right) s^{2}+16 \alpha_{1}^{2}- \\
16=0 .
\end{gathered}
$$

If the Poisson's ratio of the half space is 40 known this can be solved numerically and used to find the Rayleigh wave speed, with the following

$$
s=\frac{k_{\mathrm{T}}}{k}=\frac{\omega}{k C_{\mathrm{T}}} .
$$

45 The Rayleigh wave speed has been defined as $C_{\mathrm{R}}=\omega / k$, meaning that $s=C_{\mathrm{R}} / C_{\mathrm{T}}$. The velocity of propagation is thus independent of the frequency and the waves are therefore non dispersive. The 50 velocity of the wave depends solely on the elastic constants of the material. The rate at which the wave amplitude reduces with the depth $z$ depends on the values of the factors $\gamma_{\mathrm{L}}$ and $\gamma_{\mathrm{T}}$ which are given by:

$$
\frac{\gamma_{\mathrm{L}}^{2}}{k^{2}}=1-\alpha_{1}^{2} s^{2}
$$

$$
\frac{\gamma_{\mathrm{T}}^{2}}{k^{2}}=1-s^{2}
$$

If $v>0.263$ there are two complex conjugate roots and one real root. If ${ }_{60} v \leq 0.263$ then there will be three real roots $^{6}$. However, only one of these roots will be realistic. The change of amplitude with depth for a Rayleigh wave can be calculated from:

$$
\begin{gathered}
u_{\mathrm{R}}=\frac{\partial \varphi}{\partial x}+\frac{\partial \psi}{\partial z}= \\
-\left(A \mathrm{i} k \mathrm{e}^{-\gamma_{\mathrm{LZ}}}+B \gamma_{\mathrm{T}} \mathrm{e}^{-\gamma_{\mathrm{TZ}}}\right) \mathrm{e}^{[\mathrm{i}(\omega t-k x)]},
\end{gathered}
$$

$$
\begin{gathered}
w_{\mathrm{R}}=\frac{\partial \varphi}{\partial z}-\frac{\partial \psi}{\partial x}= \\
-\left(A \gamma_{\mathrm{L}} e^{-\gamma_{\mathrm{L} Z}}-B \mathrm{i} k e^{-\gamma_{\mathrm{T} Z}}\right) e^{[\mathrm{i}(\omega t-k x)]} .
\end{gathered}
$$

70 These reduce to the following if the real parts are taken and $B$ is substituted for $A$ :

$$
\begin{gathered}
u_{\mathrm{R}}=A k f_{u(z)} \sin (\omega t-k x), \\
w_{\mathrm{R}}=A \gamma_{\mathrm{L}} f_{w(z)} \cos (\omega t-k x) .
\end{gathered}
$$

where we have introduced the decay 75 functions $f_{u(z)}$ and $f_{w(z)}$ given by

$$
\begin{aligned}
& f_{u(z)}=e^{-\gamma_{\mathrm{LZ}}}-\frac{2 \gamma_{\mathrm{L}} \gamma_{\mathrm{T}}}{\left(\gamma_{\mathrm{T}}^{2}+k^{2}\right)} e^{-\gamma_{\mathrm{TZ}},}, \\
& f_{w(z)}=e^{-\gamma_{\mathrm{LZ}}}-\frac{2 k^{2}}{\left(\gamma_{\mathrm{T}}^{2}+k^{2}\right)} e^{-\gamma_{\mathrm{TR} Z}},
\end{aligned}
$$

which describe the rate at which the 80 displacement amplitudes along the direction of propagation change with depth. Displacement in the $x$ (horizontal) direction decreases rapidly as $z$ increases; this can be seen in Figure 2(a) which 85 shows an example of displacements as a function of depth. The functions are normalised against the amplitude of the motion perpendicular to the propagation direction at the surface. At a depth of 90 about $z / \lambda=0.2$ the displacement in the $x$ direction passes through zero, and changes polarity. Movement perpendicular to the surface, i.e. in the $z$ direction, increases slightly before 95 reaching a maximum at a depth of about 0.076 wavelengths and then falls away but does not change polarity.

From Eqs (28a) and (28b), it can be seen that the displacements $u$ and $w$, are 100 functions of sine and cosine respectively. Hence the motion of particles is rotational about the $y$ axis, rather than traversing back and forth along a curved path. This can be seen in 105 Figure 2(b) which shows the paths of particles (arbitrary amplitude) at depths corresponding to Figure 2(a).

Particle motion can be thought of as planes of elliptical motion, whose shape 110 and phase at any given time depends upon the depth. The motion is anticlockwise near the surface but reverses to a clockwise motion below the zero crossing of the $u$ velocity 115 component. An alternative, simpler 
approximation for the speed of Rayleigh waves (i.e. the root of Eq.(24)), which expresses $s$ solely in terms of Poisson's ratio, is quoted by Victorov ${ }^{7}$ :

$$
s=\frac{0.87+1.12 v}{1+v} .
$$

5

Thus as $v$ varies from 0 up to $0.5, C_{\mathrm{R}}$ varies from $0.87 C_{T}$ to $0.96 C_{T}$. This is shown in Figure 3.

It is worth noting that actual 10 displacements are very small. As an example $^{8}$, in a device operating at $100 \mathrm{MHz}$ with $10 \mathrm{~mW}$ average power in a beam $1 \mathrm{~cm}$ wide on a substrate with SAW velocity $3 \mathrm{~km} \mathrm{~s}^{-1}$, the wavelength is $1530 \mu \mathrm{m}$ and the peak vertical displacement of the order of $10^{-10} \mathrm{~m}$.

This section has discussed the mechanism of surface wave propagation and the following section describes more general 20 conditions which are of importance to ultrasonic particle manipulation.

\section{Leaky Rayleigh Waves}

A pure Rayleigh wave (with a completely free surface) is of less interest in 25 microfluidics than a surface bounded by a fluid or solid into which the surface wave will deliver energy. This will lead to an exponentially decaying wave propagating along the fluid-solid interface ${ }^{9}$. In practice 30 this attenuation is relatively small for solid-gas interfaces. In the following a fluid loaded structure is considered. The fluid is assumed to be unbound. As the impedance of the fluid rises from zero, 35 the wave will behave less like a pure Rayleigh wave and will transform into a "leaky" Rayleigh wave.

The components of a Rayleigh wave can be broken down into normal and 40 tangential displacements and arranged into the following form ${ }^{10}$ :

$$
4 k^{2} \gamma_{\mathrm{L}} \gamma_{\mathrm{T}}-\left(k^{2}+\gamma_{\mathrm{T}}^{2}\right)^{2}=0 .
$$

When the surface is loaded with a fluid, 45 the boundary conditions change. By including the continuity of normal stress into the above equation the following equation is generated:

$$
4 k^{2} \gamma_{\mathrm{L}} \gamma_{\mathrm{T}}-\left(k^{2}+\gamma_{\mathrm{T}}^{2}\right)^{2}=\mathrm{i} \frac{\rho_{F}}{\rho_{\mathrm{R}}} \frac{\gamma_{\mathrm{L}} k_{\mathrm{T}}^{4}}{\sqrt{k_{\mathrm{L}}^{2}-k^{2}}},
$$

where $\rho_{\mathrm{F}}$ and $\rho_{\mathrm{R}}$ are the densities of the fluid and the solid half space respectively. Likewise, $C_{F}$ and $C_{R}$ are the wave speeds of the two media. Tangential ${ }_{55}$ displacement is assumed not to be transferred to the fluid, since it does not support shear modes but more information on this matter can be found in a recent paper by Vanneste \& Bühler ${ }^{11}$. ${ }_{60}$ Upon inspection it can be seen that as $\rho_{\mathrm{F}}$ tends to zero, the equation reverts back to a pure Rayleigh wave. This equation has one real root and one complex root. The real root corresponds to a Scholte wave 65 and will be discussed later. The complex root corresponds to a modified Rayleigh wave. It can be shown that given the condition $C_{\mathrm{F}}<C_{\mathrm{R}}$, which is true for most media, then Eq.(32) has a complex root 70 that corresponds to a system of three waves $^{12}$. One wave is found in the fluid and two in the solid. A simple physical interpretation of this is that the Rayleigh wave radiates energy at an angle into the 75 fluid. This must be the case since we are dealing with a complex root, which must be losing energy, and this is the radiation away from the boundary into the fluid ${ }^{10}$. It can be shown that the velocity of the 80 loaded Rayleigh wave is higher than that of the unloaded wave ${ }^{7}$. However, upon further analysis the difference can be shown to be very small. As the fluid has little effect on the velocity of the ${ }_{85}$ Rayleigh wave, the following approximation (essentially a statement of Snell's law) can be made about the angle at which the wave propagates into the material, see figure 4 :

90

$$
\sin \theta=\frac{C_{\mathrm{F}}}{C_{\mathrm{R}}} .
$$

This is found by phase matching the wave in the fluid with the Rayleigh wave. As the wave travels along the interface it can
95 be seen to "leak" energy into the fluid, at this angle. For common gases such as air this is near normal to the surface. It should be noted that a Rayleigh wave can be generated on the surface of a substrate 100 if a wave travels through the fluid at the correct angle towards the interface.

In contrast to velocity, the attenuation of the wave is greatly affected by the presence of the fluid. It is this 105 characteristic which makes Rayleigh waves excellent choices for the efficient transfer of energy into cavities or droplets. The attenuation coefficient for a leaky Rayleigh wave travelling along a 110 solid-fluid interface is given by ${ }^{10}$

$$
\alpha_{\mathrm{L}}=\frac{\rho_{\mathrm{F}} C_{\mathrm{F}}}{\rho_{\mathrm{R}} C_{\mathrm{R}} \lambda_{\mathrm{R}}} \mathrm{m}^{-1},
$$

where $\lambda_{\mathrm{R}}$ is the Rayleigh wavelength. The coefficient defines the energy loss 115 resulting from the transmission of a bulk wave into the fluid over a distance $x$ as being $\mathrm{e}^{-\alpha_{L} x}$. The attenuation of the surface wave per wavelength travelled is proportional to the ratio of the fluid and 120 solid impedances. The attenuation coefficient is relatively small for atmospheric air, so for most practical purposes can be ignored $^{13}$.

So far it has been assumed that the 125 attenuation of the leaky Rayleigh wave is due to the radiation of a compression wave into the fluid; however there will also be frictional losses from the transverse motion of the surface. This can 130 be given by the following equation ${ }^{10}$ :

$$
\alpha_{\mathrm{s}}=\frac{\left(\rho_{\mathrm{F}} \eta \frac{\omega^{3}}{2}\right)^{\frac{1}{2}}}{4 \pi^{2} \rho_{\mathrm{R} C \mathrm{R}}^{2}} \mathrm{~m}^{-1}
$$

Where $\eta$ is the viscosity of the medium and $\omega$ is the angular frequency of the leaky Rayleigh wave. These forces are 135 typically small compared to the longitudinal contribution to attenuation. Typical calculated attenuation factors for leaky Rayleigh waves in $\mathrm{LiNbO}_{3}$ are shown in Table 1. 


\section{Cite this: DOI: $10.1039 / \mathrm{c} 0 x \times 00000 x$}

www.rsc.org/loc

FOCUS

Table 1 Calculated values for the attenuation of Rayleigh waves in lithium niobate by ambient media

\begin{tabular}{|c|c|c|c|c|}
\hline \multirow[b]{2}{*}{ Constant } & \multirow[b]{2}{*}{ Medium } & \multicolumn{3}{|c|}{ Attenuation coefficient $\left(\mathrm{m}^{-1}\right)$. Bracketed values in $\mathrm{dB} / \mathrm{cm}$} \\
\hline & & $10 \mathrm{MHz}$ & $100 \mathrm{MHz}$ & $300 \mathrm{MHz}$ \\
\hline$\alpha_{\mathrm{L}}^{a}$ & Atmospheric Air & $7.4 \times 10^{-2}(0.0032)$ & $0.74(0.032)$ & $2.2(0.096)$ \\
\hline$\alpha_{\mathrm{L}}{ }^{a}$ & Water & 260 & $2600(110)$ & $7800(330)$ \\
\hline$\alpha_{\mathrm{s}}{ }^{b}$ & Water & $0.16 \quad(0.0067)$ & $5.0(0.21)$ & $26(1.1)$ \\
\hline
\end{tabular}

${ }^{a}$ Attenuation due to radiation of longitudinal waves.

${ }^{b}$ Attenuation due to frictional loss

From Table 1 it can be seen the 5 longitudinal component of attenuation increases linearly with frequency and that in air this is negligible for all frequencies. The frictional component of attenuation increases proportionally with the angular 10 frequency to the power of 1.5 and is negligible except for extremely high frequencies, which are not reached in the particle and droplet manipulation devices mentioned later.

\section{D. Scholte Waves}

As mentioned earlier, there is a real solution to Eq.(32). Further analysis shows that it is quite different from a Rayleigh wave and that such solutions 20 exist for any combination of fluid and solid $^{14}$. It can be shown that the wave corresponds to a surface wave travelling along the boundary with a velocity less than the wave velocity of the fluid and the

25 longitudinal and transverse wave speeds of the solid ${ }^{15}$. For lossless media they do not attenuate and simply propagate along the interface: they are therefore pure interface waves. For fluids where the 30 acoustic impedance is less than that of the solid, decrease in displacement amplitude with depth is significantly higher in the solid than the fluid and most of the energy is contained within the fluid, in 35 contrast to the leaky Rayleigh wave ${ }^{7}$. Scholte wave generation efficiency increases as the acoustic impedance of the fluid increases so little Scholte wave generation is seen when air is the fluid.

${ }_{40}$ As the impedance of the fluid increases compared to the solid, the Scholte wave penetrates deeper into the solid ${ }^{16}$.

\section{E. Interface Waves}

${ }_{45}$ All waves described so far have propagated along a solid-vacuum or solidfluid interface. A solid-solid interface will now be considered. For the purpose of this discussion only Rayleigh-like modes 50 will be considered. Love modes, with motion perpendicular to the direction of propagation are currently of little interest for ultrasonic particle manipulation.

${ }_{55}$ The addition of a thin layer (thickness $h$ ) on a substrate carrying a Rayleigh wave provides geometric constraints which will alter the characteristics of propagation, making the wave dispersive. This ${ }_{60}$ property has been used to great effect for SAW devices, as particular dispersion characteristics can be designed for ${ }^{4}$. As the layer thickness tends to zero, the wave transforms into a standard Rayleigh wave.

${ }_{65}$ The added thin layer can have several different effects on the wave velocity. The following inequality can be used to predict the behaviour of the dispersion curve as $k h$ tends to zero ${ }^{17}$ 70

$$
\frac{C_{\mathrm{T} 2}}{C_{\mathrm{T} 1}}>\sqrt{\frac{1-\left(\frac{C_{\mathrm{T} 1}}{C_{\mathrm{L} 1}}\right)^{2}}{1-\left(\frac{C_{\mathrm{T} 2}}{C_{\mathrm{L} 2}}\right)^{2}}}
$$

where subscripts 1 and 2 represent the substrate and added layer respectively, whilst ${ }_{\mathrm{T}}$ and ${ }_{\mathrm{L}}$ represent transverse and 75 longitudinal as before. The limits of the right hand side are $\sqrt{2}$ and $1 / \sqrt{2}$. If the above inequality is true the dispersion curve will be positive as the layer thickness approaches zero. If the above 80 inequality is false, the dispersion curve will have a negative gradient as the layer thickness approaches zero. The case in which the solution lies between these boundaries is more difficult and shall be 85 covered later. This inequality can be presented using shear moduli and density ratios as the ordinate and abscissa axis respectively, see Figure $5^{4}$.

Should the inequality be true, then the 90 layer is said to stiffen the substrate because the presence of the substrate leads to an increase in the surface wave velocity above the unloaded Rayleigh wave velocity. As the layer thickness 95 increases the wave speed increases monotonically up to the shear velocity of the substrate.

As $k h$ increases further, the wave leaks 100 into the substrate so it is no longer a true interface wave. Therefore Rayleigh type propagation is only possible for small values of $k h$. The variation in wave speed is minimal for these material parameters, 105 as it is dominated by the substrate material parameters. It should be noted that this set of material conditions is an unlikely scenario for ultrasonic particle manipulation devices.

110 Should $C_{\mathrm{T} 2}<C_{\mathrm{T} 1} / \sqrt{2}$ then the layer loads the substrate and the phase velocity of the Rayleigh-like mode decreases as $k h$ increases, until it approaches the Rayleigh velocity of the layer. This is for a layer 115 thickness large in comparison to the wavelength. At higher values of $k h$, the group velocity and phase velocity converge, as characteristics imposed by the layer are less important with regards 120 to the wavelength. The interface waves 
described so far radiate energy away from the interface.

\section{F. Stoneley Waves}

There exists a particular set of conditions 5 such that a Rayleigh-like wave can propagate at the interface between two solids; this type of wave is called a Stoneley wave which is evanescent in both media ${ }^{4}$. For a Stoneley wave to exist 10 both the longitudinal and transverse displacements must be transferred in such a way that energy is confined to the interface. The derivation of a Stoneley wave is similar to that of a Rayleigh

15 wave, except for the transmission of displacement and stress across the boundary. The velocity of the Stoneley wave must be less than the transverse wave speeds of both media but more than 20 the Rayleigh wave speed of the denser medium. The transverse wave speeds of both media need to be similar for Stoneley waves to exist. Stoneley wave velocity is not dependent on frequency, 25 so the wave is therefore non dispersive. Stoneley waves do not attenuate along the path of propagation so are ideal for the efficient transfer of energy into a sealed fluid chamber.

\section{${ }_{30}$ G. Anisotropic media and piezoelectric considerations}

The description so far has been based on isotropic non-piezoelectrically active materials. Most materials used for surface 35 acoustic wave devices are anisotropic and in many cases single crystal. The Rayleigh wave and associated Rayleighlike waves still exist for any given direction of propagation but the 40 characteristics will differ based on orientation. $\mathrm{LiNbO}_{3}$ is often used and it has a typical Rayleigh wave speed between $3500 \mathrm{~m} \mathrm{~s}^{-1}$ and $4000 \mathrm{~m} \mathrm{~s}^{-1}$ depending upon orientation. Depending 45 on the materials used, the ellipse of motion need not be normal to the surface and displacement in the $x$ and $z$ direction may oscillate with depth $^{4},{ }^{18}$. Wave propagation in anisotropic materials is ${ }_{50}$ less straightforward than isotropic materials however, gains can be made in piezoelectric coupling and the correct orientation will suppress other undesirable wave modes ${ }^{4}$. In an 55 anisotropic solid there are two different transverse modes as well as the longitudinal mode, and as a result a more general surface wave is produced with displacements in three dimensions. This 60 type of wave can be regarded as a combination of Rayleigh and Love waves. It is possible to find orientations that decouple these waves into their respective pure Rayleigh and Love 65 components ${ }^{19}$.

\section{H. Generation of surface acoustic waves}

There are several methods of generating surface waves. A transducer coupled 70 through a wedge can be designed using the inverse of Eq.(33), see Figure 6.

In this case the Rayleigh wave generated will propagate in one direction only and the coupling angle is independent of the 75 required frequency ${ }^{6}$. The wedge material must have a low longitudinal wave speed, as for this to work, requiring that $C_{\mathrm{W}}<C_{\mathrm{R}}$. Alternatively a periodic transducer array, with contacts coupling ${ }_{80}$ to the surface at a spacing of $\lambda_{R}$ can be used, this will radiate both to the left and the right, see Figure 7.

These transduction methods rely on coupling the energy from an active 85 transducer into a passive substrate, but the most common means of generating SAWs is to use a piezoelectrically active substrate or a thin piezoelectric layer on a substrate. In such a case the excitation 90 (and reception) of the SAW is provided by sets of interdigital transducers (IDTs) which comprise metallic strips deposited directly onto the piezoelectric surface. Figure 8 shows a simple IDT electrode ${ }_{95}$ pattern with two sets of interleaved combs extending from the two electrode rails. When a signal is applied to these rails an electric field is established with the spatial period of the comb. This then 100 excites a strain field via piezoelectric coupling. The response will be strongest when the spatial period of the electrodes equals the SAW wavelength in the substrate. For a single electrode pair the ${ }_{105} \mathrm{Q}$ factor is small and the response is broadband, but as more identically spaced electrode pairs are added, the $\mathrm{Q}$ factor increases and the IDT develops a sharp resonance peak. Such an IDT will 110 generate a SAW that will propagate in both directions. If designing for a wave that travels in one direction only attention must be given to absorbing (or reflecting) the opposite-going wave. 115

A number of additional practical considerations need to be taken into account when designing SAW systems, including the facts that: temperature 120 effects may be significant and may also vary with orientation, and models for SAW propagation in passive substrates do not fully describe SAW behaviour in a piezoelectric substrate as the 125 electromechanical coupling complicates the material's mechanical characteristics. When generating waves it is important to note that electrodes themselves modify the impedance presented to the SAW, 130 through mechanical loading and modification of the electromechanical coupling in the substrate adjacent to the electrode. For this reason each electrode finger can be broken down into two, 135 maintaining the periodicity in terms of the electric field but breaking the periodicity in terms of reflection characteristics. It is normal for the spacing between fingers to be of equal width to the electrodes. This 140 means they are $\lambda / 4$ for single electrode configurations or $\lambda / 8$ for double electrode configurations. Devices for ultrasonic particle manipulation tend to work at frequencies of $20-40 \mathrm{MHz}$ which 145 corresponds to electrode widths of approximately $50-25 \mu \mathrm{m}$ for a typical $\mathrm{LiNbO}_{3}$ device. Most devices are no bigger than a standard microscope glass slide. Electrode fingers need not be 150 parallel to each other ${ }^{20,21}$ and can even be concentrically curved to allow for tight focussing into a point on the surface ${ }^{22}$.

\section{Applications of SAW devices}

The most common uses for SAW devices 155 are for electronic filter applications and in sensors, but this section will concentrate on a few examples of applications in microfluidic devices. See Yeo and Friend or Wang and Zhe for more 160 comprehensive reviews ${ }^{23}, 24$.

\section{Planar Microfluidics}

A well-known use of surface acoustic waves is in planar microfluidics ${ }^{25,} 26$, 165 which differs from the sealed chamber devices of particular interest in this work These devices allow droplets to be manipulated on the surface of a piezoelectric substrate and have the 170 potential to overcome many of the problems of priming and clogging 
reported in channel-based microfluidics. The droplets are driven by their interaction with SAWs travelling through the substrate, and modification of the 5 surface wetting properties allows further control of the droplet trajectory.

If a surface acoustic wave is projected towards a droplet, it will become a leaky 10 Rayleigh wave upon contact with the droplet, transferring energy into the fluid at a calculable angle. This generates streaming within the droplet which will distort its shape. At sufficiently high 15 amplitudes this distortion alters the contact angle of the droplet with the surface and this propels it along the substrate in the direction of the wave $\mathrm{e}^{25,27}$. This can, in turn, be used to move 20 droplets of different reagents, to split droplets into smaller droplets, and to merge and mix reagents. This has been used in a microfluidic PCR (Polymerase Chain Reaction) DNA amplification 25 device in which the reagent droplets, covered with an oil film to control evaporation, are cycled between heating stages and mixed using $\mathrm{SAWs}^{28}$. Other reported applications of planar 30 microfluidics include the concentration of particle samples within droplets ${ }^{29}$, concentration and mixing using focussed $\mathrm{SAWs}^{30}$ and the use of SAWs within a PDMS channel on a $\mathrm{LiNbO}_{3}$ substrate as 35 an actuation mechanism within a twoway droplet sorting scheme ${ }^{31}$ as shown schematically in Figure 9. A related planar device, in which the fluid volume is constrained by a cover slip, has been 40 used to investigate mixing induced by SAWs in microfluidic volumes ${ }^{21}$.

\section{SAW induced flow in channels}

SAWs can initiate mixing within 45 enclosed fluid volumes as well as in droplets on a substrate. Mixing is a critical issue in microfluidic systems as the very low Reynolds numbers mean that mixing processes tend to be diffusion 50 limited and slow. The mechanism that allows SAWs to induce mixing is generally that of acoustic streaming induced within the fluid. A droplet mixing technique has been used to induce 55 mixing in PDMS channels coupled to a $\mathrm{LiNbO}_{3}$ substrate ${ }^{32}$. The channels were $75 \times 100 \mu \mathrm{m}$ and were excited at a frequency of $146 \mathrm{MHz}$. Effective mixing was reached within one tenth of the
60 distance required without SAW excitation. The authors note the tendency of the system to trap and aggregate particles during the mixing process although the cause of the phenomenon is 65 not discussed in detail.

Surface acoustic waves can be used to induce bulk flows and/or mixing in similar devices, depending on the width of the fluid channel compared to the 70 excitation wavelength ${ }^{33}$. Tan et al. report that when the wavelength was greater than the channel width the predominant effect was bulk flow, or a pumping action, while for larger channel widths, 75 mixing vortices predominate.

Other applications of SAW excitation to induce flow in microfluidics include: the generation of streaming to accelerate mass transfer for reagent uptake ${ }^{34}$; the 80 disruption and removal of bio-films ${ }^{35}$; the release of non-specifically bound proteins from biosensor surfaces ${ }^{36}$; the transport of cells into tissue scaffolds to enhance seeding for tissue engineering 85 applications $^{37,38}$; and the investigation of cell adhesion properties ${ }^{39}$.

SAWs for radiation force particle
manipulation

${ }_{90}$ Surface acoustic waves can also be used to generate acoustic energy gradients for ultrasonic particle manipulation. Standing waves can be used to align particles in bands within droplets ${ }^{40}$ and at points in 95 sealed chambers, with or without flow ${ }^{41}$, 42 (Figure 10).

Another operation that can be carried out in flow-through fluid chambers is the tight focussing of a beam (or multiple 100 beams) in a PDMS fluid channels ${ }^{41,43,44}$.

Surface acoustic waves were excited using IDT's either side of a fluid channel. The resulting standing wave pattern has a potential well at the PDMS channel 105 centre, causing particles to be focussed within that potential well, Figure 11.

Higher frequencies result in tighter bound beams, however there is a limit to how 110 high a frequency can be used in a given channel before particles will collect at channel walls, which for many applications is undesirable. A similar device can be used to separate particles of 115 different sizes, as the forces exerted on a particle are proportional to its volume $\left(r^{3}\right)$, but viscous drag is only proportional to particle radius $(r)$ leaving a sufficiently large difference for particle 120 separation. This results in fractionation based on the time taken for the particle to move from its starting position to its end position.

Similar techniques could be used to 125 separate particles based on density or compressibility, but this has not yet been shown in a surface acoustic wave device. This technology could also be used to separate particles with positive and 130 negative acoustic contrast factor, which has been demonstrated in a bulk acoustic device ${ }^{45}$.

Improvements in particle focussing can be made through limiting the amount of ${ }_{135}$ PDMS the Rayleigh wave has to travel through before it reaches the fluid channel and by incorporating Bragg reflectors ${ }^{46}$. Particle position within the fluid channel can be altered using a phase 140 shifting technique ${ }^{47}$. Particle position across the channel is relatively linear with phase shift allowing for particle alignment at any position across the fluid channel.

145

Stoneley waves for radiation force particle manipulation

The use of lithium niobate with PDMS fluid channels is inherently an inefficient 150 mechanism for coupling energy into a fluid channel. There will be significant leakage of energy away from the interface into the PDMS. Further, ultrasonic propagation in PDMS exhibits relatively 155 high losses, so energy leaking into the PDMS will be rapidly attenuated. It is possible to design a manipulator using material combinations that allow for the propagation of a Stoneley wave, so losses 160 will be minimal until the wave reaches the fluid $^{48}$. By designing a high frequency and efficient device, Yanchev et al. ${ }^{48}$ demonstrated that it was possible to manipulate particles of $0.5 \mu \mathrm{m}$ diameter.

\section{${ }_{165}$ Conclusions}

This tutorial paper has derived the equations governing surface acoustic waves in their most basic solid-vacuum state before describing the general 170 behaviour of pure Rayleigh waves. The work has also shown how Rayleigh waves differ when propagating along a solid-fluid interface. The leakey SAWs generated from such an interface are 175 described and the associated losses are shown with examples. Scholte waves 
have been discussed before moving onto solid-solid interfaces and the effects this has upon wave propagation velocities. Stonely waves and their potential for 5 efficient transfer of energy along an interface are then discussed. The paper has described the more practical side of SAWs and applicable generation methods including a review of recent SAW 10 devices for ultrasonic manipulation, covering planar microfluidics, SAW induced flow and radiation force particle manipulation.

\section{Notes and references}

$15^{a}$ Address, Address, Town, Country. Fax: $X X$ XXXX XXXX; Tel: XX XXXX XXXX; E-mail: $x x x x @ a a a . b b b . c c c$

\section{${ }_{20}$ References}

1. Rayleigh, Proc. London Math. Soc., 1885, 17, 4-11.

2. H.Kolsky, Stress Waves in Solids, Clarendon Press, Oxford, 1953.

25 3. A.A.Oliner, G.W.Farnell, H.M.Gerard, E.A.Ash, J. A.J.Slobodnik and H.I.Smith, Acoustic Surface Waves, Springer-Verlag Berlin Heidelberg, 1978.

30 4. G.W.Farnell and E.L.Adler, in Physical Acoustics 9, ed. W.P.Mason and R.N.Thurston, Academic Press, New York, 1972.

5. J. Dual and T. Schwarz, Lab on a Chip, $352012, \mathbf{1 2}, 244-252$.

6. J.L.Rose, Ultrasonic Waves in Solid Media, Cambridge University Press, Cambridge, 1999.

7. I.A.Viktorov, Rayleigh and Lamb Waves,

$40 \quad$ Plenum Press, New York, 1967.

8. V.M.Ristic, Principles of Acoustic Devices, John Wiley \& Sons, New York, 1983.

9. H.Überall, in Physical Acoustics 10, ed.

45 W.P.Mason and R.N.Thurston, Academic Press, New York, 1973.

10. J.D.N.Cheeke, Fundamentals and Applications of Ultrasonic Waves, CRC Press, 2002.

50 11. J. Vanneste and O. Bühler, Proceedings of the Royal Society A: Mathematical, Physical and Engineering Science, 467, 1779-1800.

12. L.M.Brekhovskikh, Waves in Layered 11 Media, Izdatelstvo "Nauka", Moscow, 1957.
13. K.Dransfield and E.Salzmann, in Physical Acoustics 7, ed. W.P.Mason and R.N.Thurston, Academic Press,

$60 \quad$ New York, 1970

14. W.M.Ewing, Elastic Waves in Layered Media, McGraw-Hill, New York, 1957

15. V.G.Gogoladze, Trudy Seismolo. Inst. 65 Acad. Nauk. USSR, 1948, 127, 2732.

16. C. Glorieux, K. Van de Rostyne, K. Nelson, W. Gao, W. Lauriks and J. Thoen, The Journal of the 70 Acoustical Society of America, 2001, 110, 1299-1306.

17. H.F.Tiersten, Journal of Applied Physics, 1969, 40, 770-789.

18. G.W.Farnell, in Physical Acoustics 6, ed. 75 W.P.Mason and R.N.Thurston, Academic Press, New York, 1970.

19. H.F.Pollard, Sound Waves in Solids, Pion Limited, London, 1977.

20. T. Franke, S. Braunmuller, L. Schmid, A. $80 \quad$ Wixforth and D. A. Weitz, Lab on a Chip, 2010, 10, 789-794.

21. T. Frommelt, M. Kostur, M. WenzelSchäfer, P. Talkner, P. Hänggi and A. Wixforth, Physical Review 85 Letters, 2008, 100, 034502.

22. T.-D. Luong, V.-N. Phan and N.-T. Nguyen, Microfluidics and Nanofluidics, 2010, 10, 619-625.

23. L. Y. Yeo and J. R. Friend, Biomicrofluidics, 2009, 3, 012002

24. Z. Wang and J. Zhe, Lab on a Chip, 2011, 11, 1280-1285.

25. A. Wixforth, C. Strobl, C. Gauer, A. Toegl, J. Scriba and Z. von Guttenberg, Analytical And Bioanalytical Chemistry, 2004, 379.

26. A.Wixforth, Superlattices and Microstructures, 2003, 33, 389396.

100 27. D. Beyssen, L. Le Brizoual, O. Elmazria and P. Alnot, Sensors and Actuators B: Chemical, 2006, 118, 380-385.

28. Z. Guttenberg, H. Muller, H. Habermuller, A. Geisbauer, J. Pipper, J. Felbel, M. Kielpinski, J. Scriba and A. Wixforth, Lab On A Chip, 2005, 5, 308-317.

29. H. Li, J. Friend and L. Yeo, Biomedical Microdevices, 2007, 9, 647-656.
30. R. Shilton, M. K. Tan, L. Y. Yeo and J. R. Friend, Journal of Applied Physics, 2008, 104, 014910-014919.

31. T. Franke, A. R. Abate, D. A. Weitz and A. Wixforth, Lab on a Chip, 2009, 9, 2625-2627.

32. K. Sritharan, C. J. Strobl, M. F. Schneider, A. Wixforth and Z. Guttenberg, Applied Physics Letters, 2006, 88 , 054102-054103.

33. M. K. Tan, L. Y. Yeo and J. R. Friend, EPL (Europhysics Letters), 2009, 87, 47003.

34. W. F. Paxton, M. J. O'Hara, S. M. Peper, 125 S. L. Petersen and J. W. Grate, Analytical Chemistry, 2008, 80, 4070-4077.

35. S. K. R. S. Sankaranarayanan, S. Cular, V. R. Bhethanabotla and B. Joseph, Physical Review E, 2008, 77, 066308.

36. R. Singh, S. K. R. S. Sankaranarayanan and V. R. Bhethanabotla, Journal of Applied Physics, 2010, 107, 024503-024509.

37. M. Bok, H. Li, L. Y. Yeo and J. R. Friend, Biotechnology and Bioengineering, 2009, 103, 387-401.

38. H. Li, J. R. Friend and L. Y. Yeo, Biomaterials, 2007, 28, 4098-4104.

39. M. A. Fallah, V. M. Myles, T. Kruger, K. Sritharan, A. Wixforth, F. Varnik, S. W. Schneider and M. F. Schneider, Biomicrofluidics, 2010, 4, 024106-024110.

40. C. D. Wood, S. D. Evans, J. E. Cunningham, R. O'Rorke, C. Walti and A. G. Davies, Applied Physics Letters, 2008, 92, 044104

150 41. J. Shi, D. Ahmed, X. Mao, S.-C. S. Lin, A Lawit and T. J. Huang, Lab on a Chip, 2009, 9, 2890-2895.

42. C. D. Wood, J. E. Cunningham, R. O'Rorke, C. Walti, E. H. Linfield, A. G. Davies and S. D. Evans, Applied Physics Letters, 2009, 94 , 054101.

43. J. Shi, H. Huang, Z. Stratton, Y. Huang and T. J. Huang, Lab on a Chip. 2009, 9, 3354-3359.

44. J. Shi, X. Mao, D. Ahmed, A. Colletti and T. J. Huang, Lab on a Chip, 2008, 8, 221-223

45. F. Petersson, A. Nilsson, C. Holm, H 165 Jonsson and T. Laurell, Lab on a Chip, 2005, 5, 20-22. 
46. Q. Zeng, H. W. L. Chan, X. Z. Zhao and Y. Chen, Microelectronic Engineering, 87, 1204-1206.

47. L. Meng, F. Cai, Z. Zhang, L. Niu, Q. Jin, F. Yan, J. Wu, Z. Wang and $\mathrm{H}$. Zheng, Biomicrofluidics, 2011, 5, 044104-044110.
48. V. Yantchev, J. Enlund, I. Katardjiev and L. Johansson, Journal of Micromechanics and Microengineering, 2010, $\mathbf{2 0}$, 035031.

15

\section{Medium}

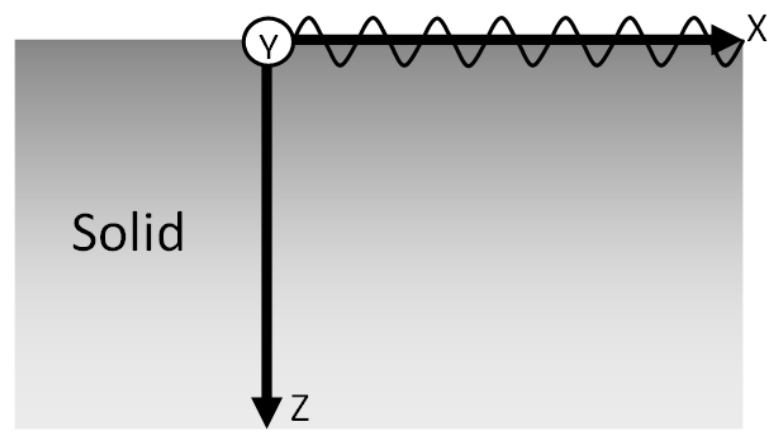

25

Figure 1. A schematic showing the propagation of a surface wave along the xy plane of a half space. 


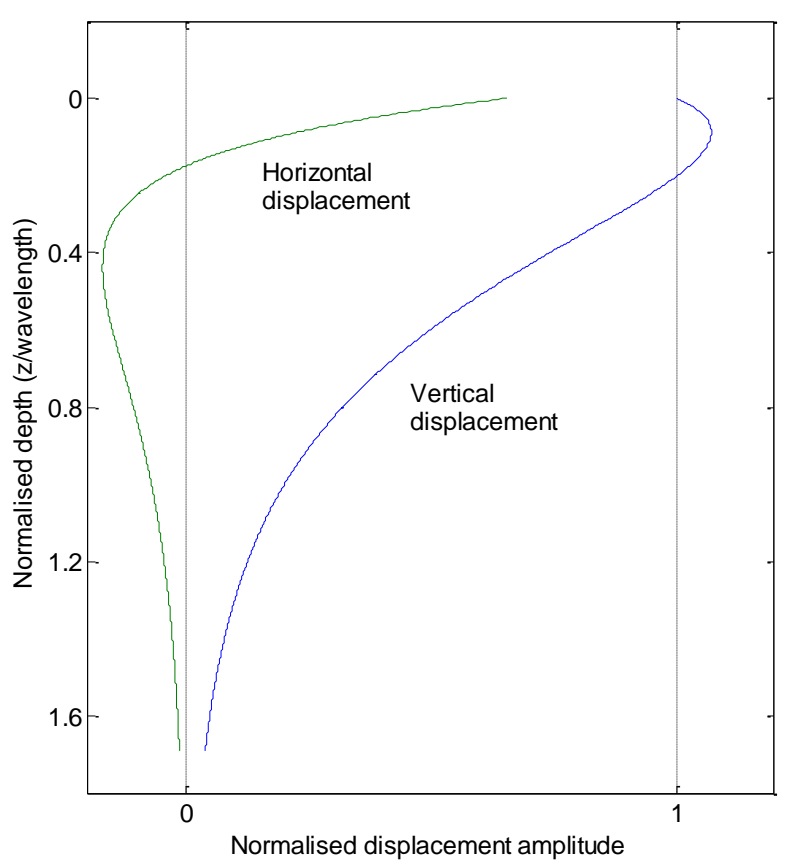

(a)

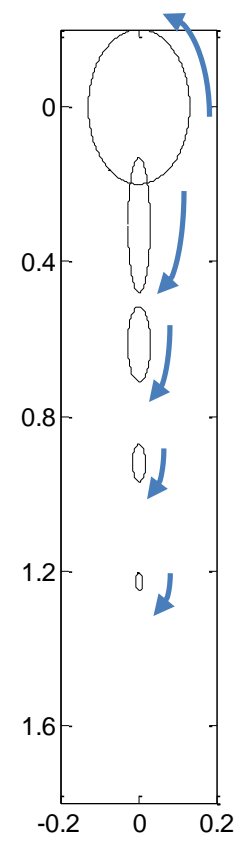

(b)

Figure 2. (a) Displacement amplitudes for a Rayleigh wave as a function of depth for an example isotropic 5 material based on equations 28. (b) Elliptical particle motions for different depths. Each ellipse corresponds to the motion of a particle with its equilibrium position at its centre. The top ellipse will rotate in the opposite direction to the lower ellipses as indicated by the blue arrows 


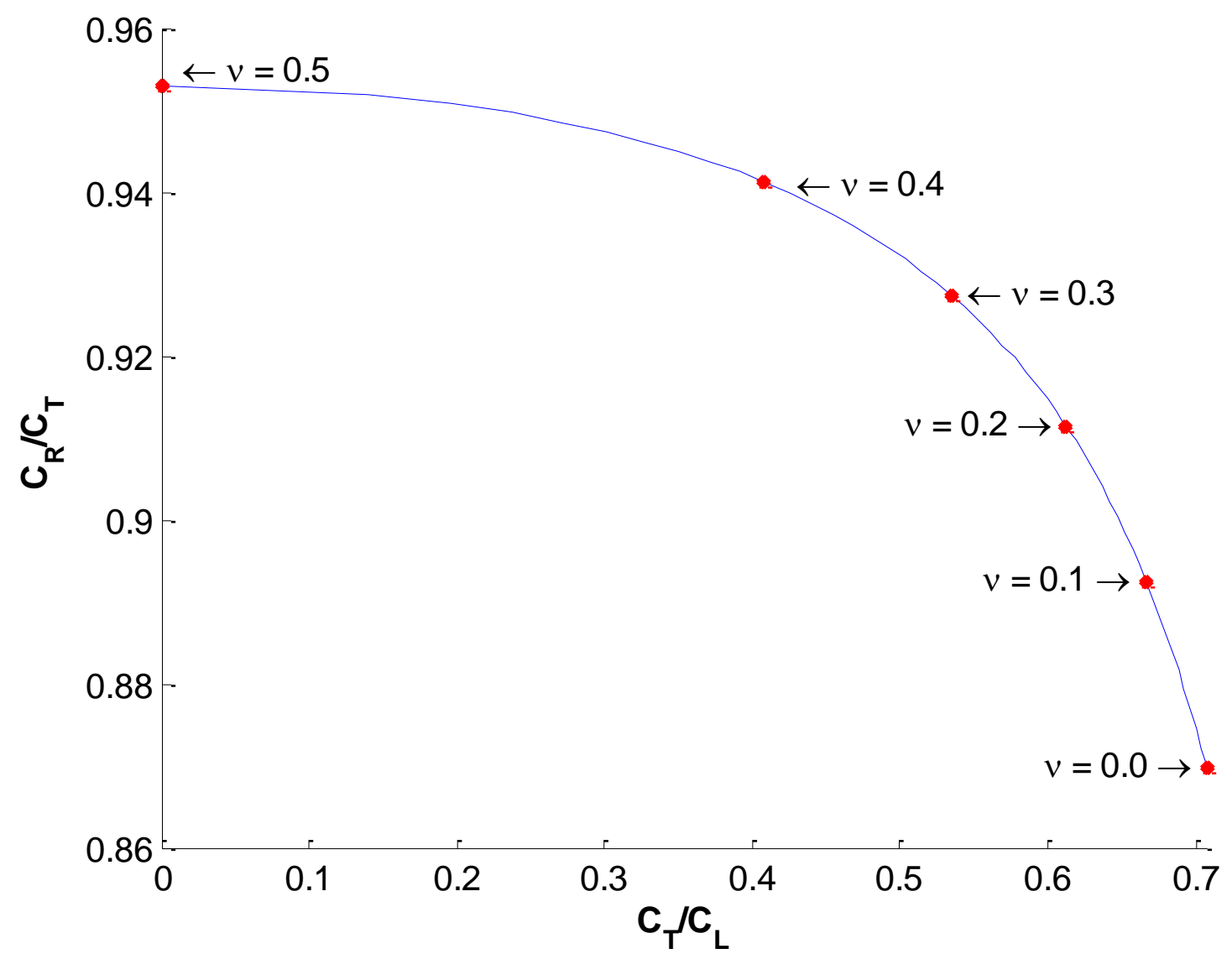

Figure $3-$ Variation in the ratios $C_{\mathrm{R}} / C_{\mathrm{T}}$ and $C_{\mathrm{T}} / C_{\mathrm{L}}$ for different Poisson's ratios $(v)^{10}$. 


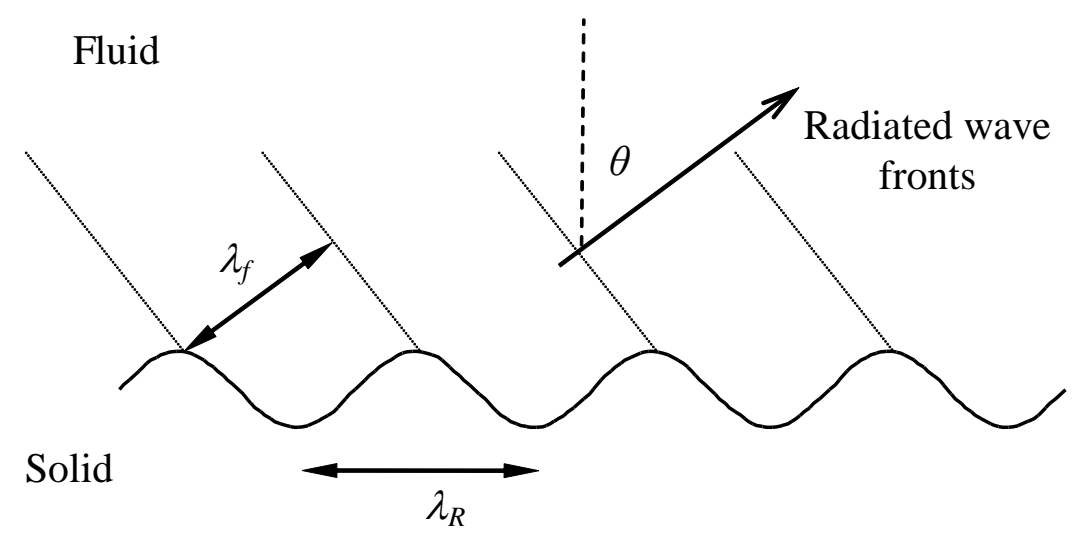

Figure 4. A schematic showing a SAW wave radiating into a fluid. 


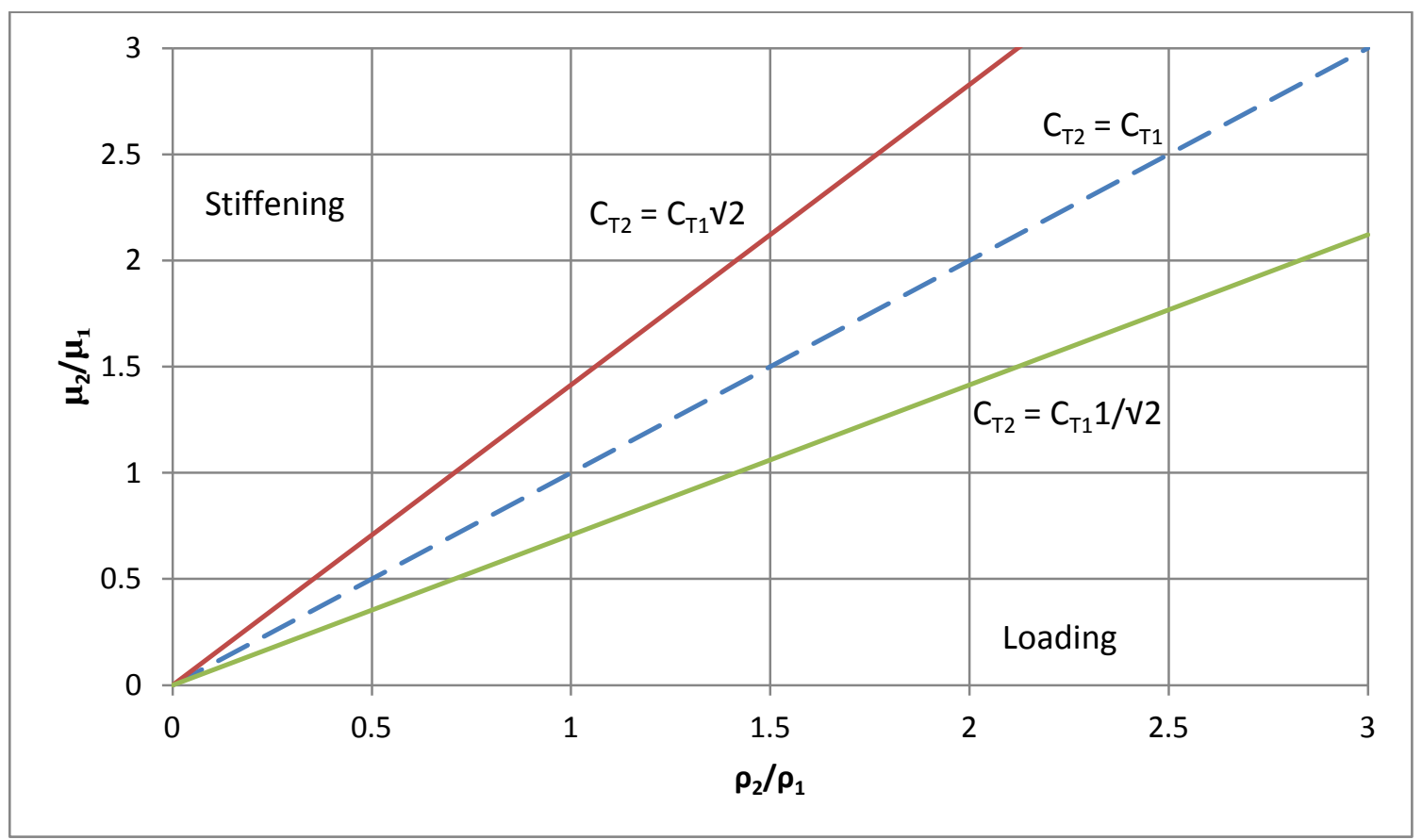

Figure 5. The limits of the inequality that determines stiffening or loading of a Rayleigh wave ${ }^{4}$. A stiffening substrate will cause an increase in the Rayleigh wave speed. A loading substrate will cause a drop in the ${ }_{5}$ Rayleigh wave speed. It is not possible to generalise on the influence of the layer on the Rayleigh wave speed if the properties lie between the two solid lines. 


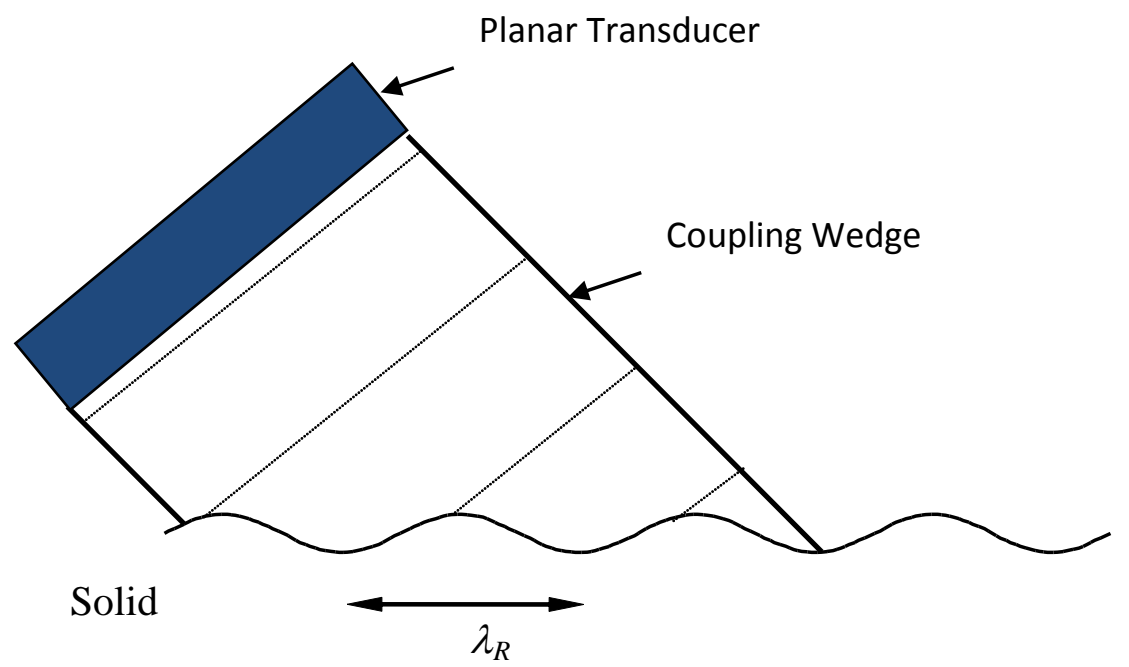

Figure 6. Wedge coupling to generate Rayleigh waves in a solid, the waves produced will be unidirectional. 


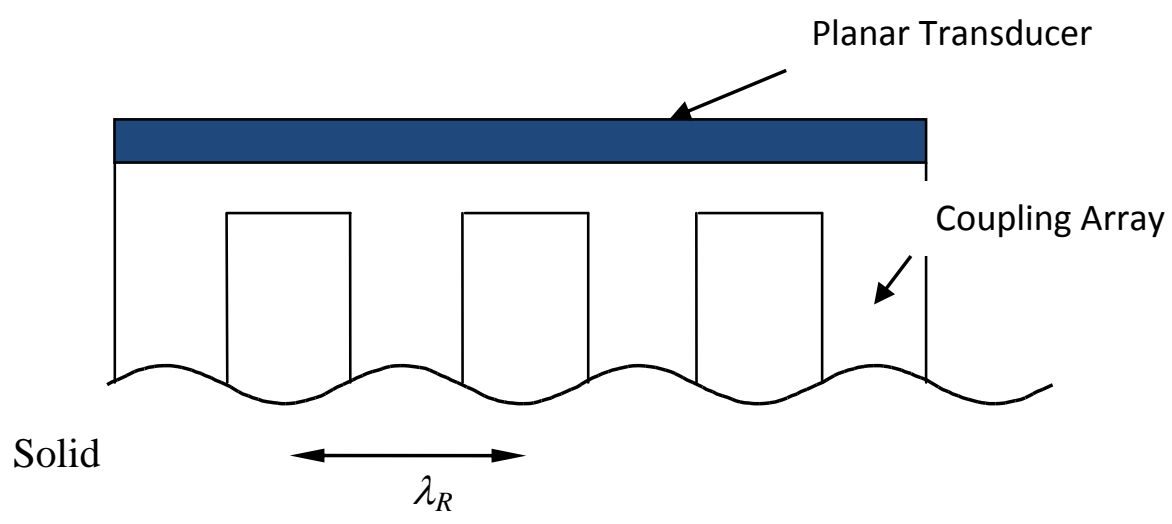

Figure 7. A periodic array for the generation of SAWs. The waves produced will be bidirectional and the frequency efficiency. The coupling array is usually made from metal'. 


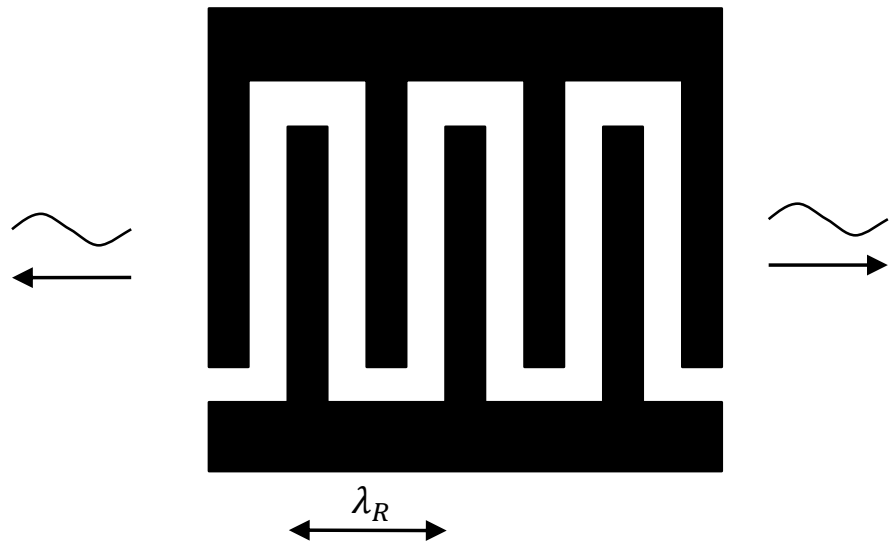

Figure 8. Uniform, metallic, periodic IDT array deposited on piezoelectric substrate. 

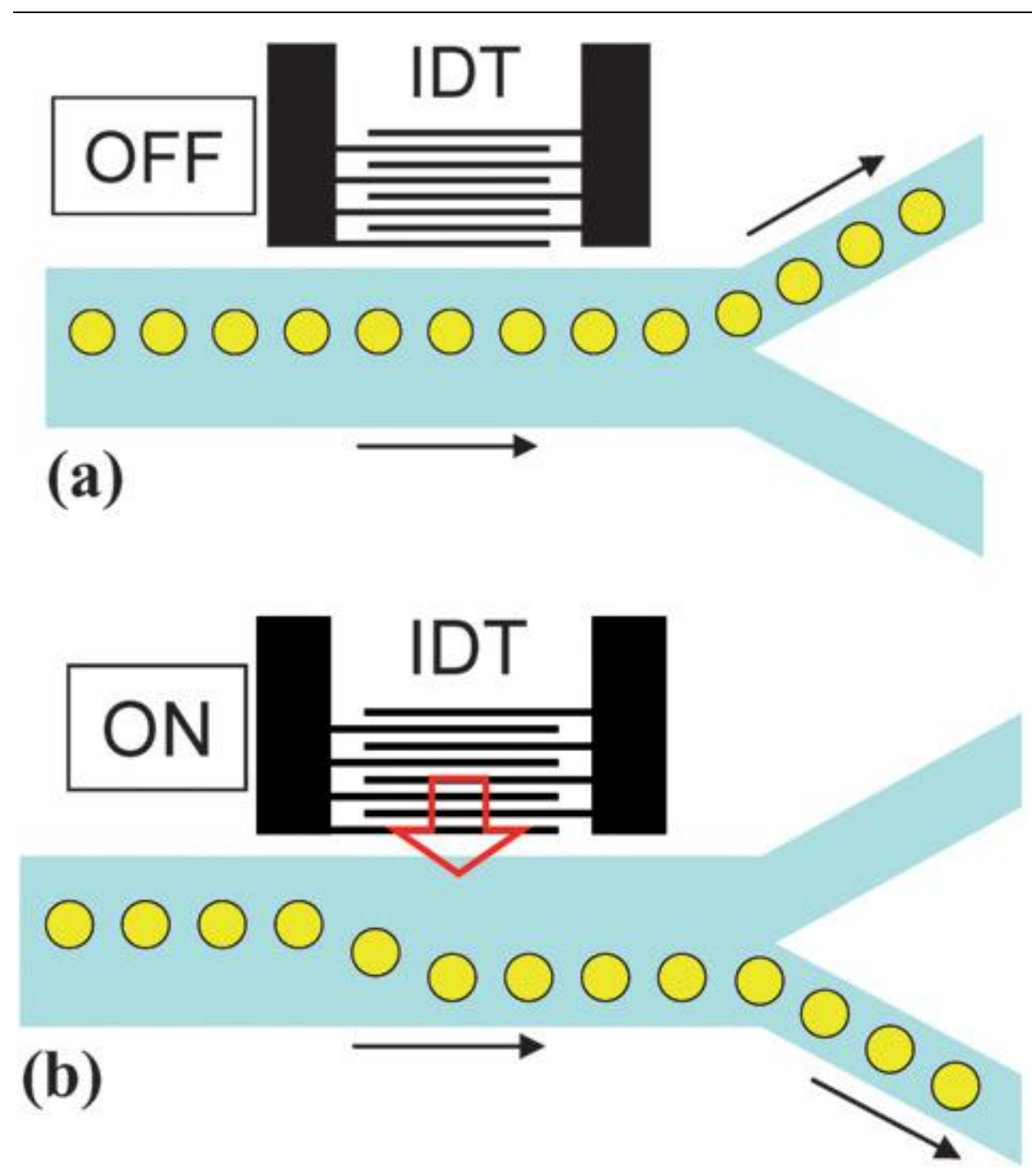

Figure 9. Schematic representation of the droplet sorting device described by Franke et al. ${ }^{31}$ (a) When the ${ }_{5}$ SAW power is off, droplets exit through the upper, lower resistance channel. (b) When the SAW is activated, the droplets are driven to the lower channel by the streaming induced by the SAW. Reproduced from Ref 31. 

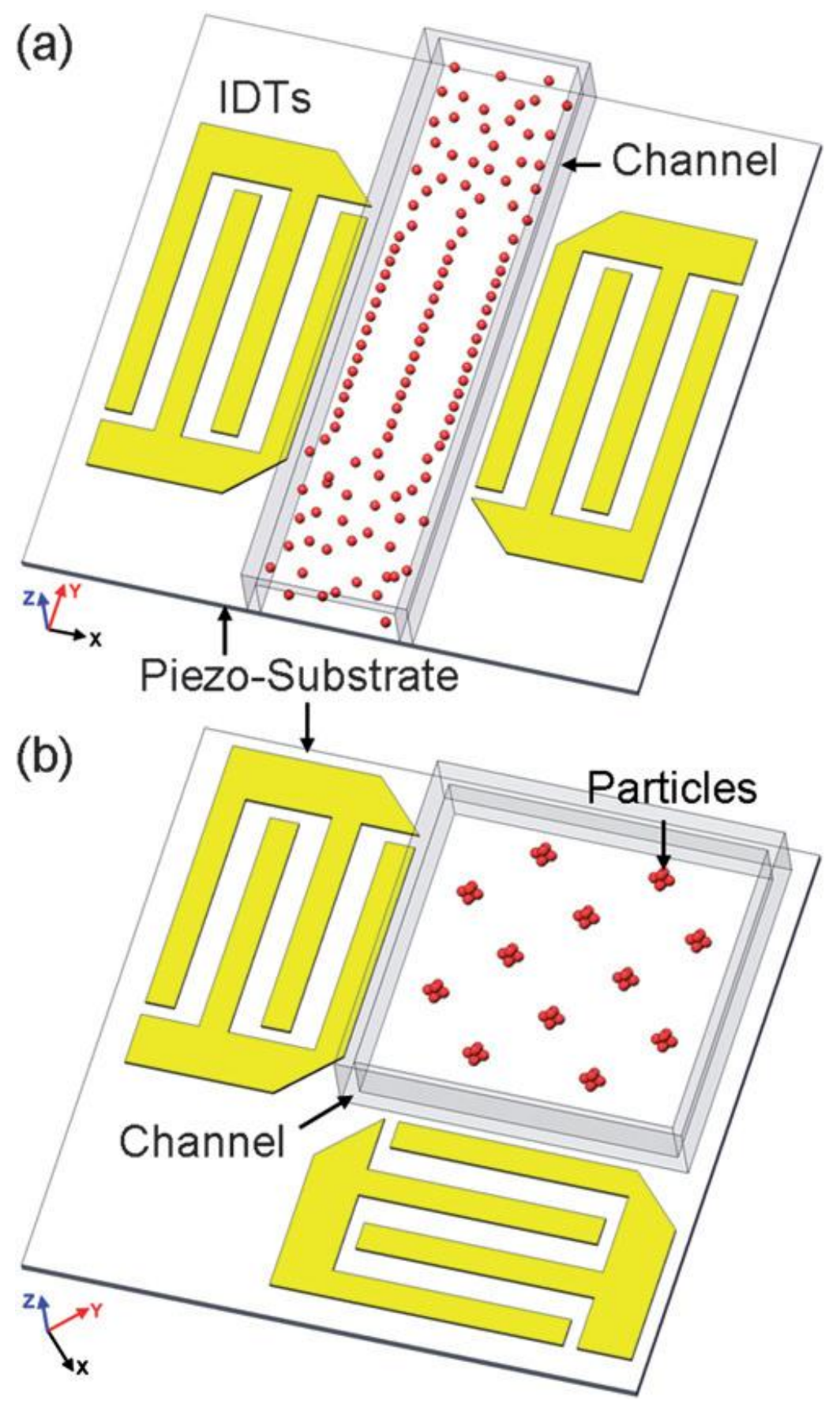

Figure 10. Particle patterning as described by Shi et al. ${ }^{41}$ (a) One dimensional patterning using a pair of opposing IDTs (b) Two dimensional patterning using orthogonal IDTs. Reproduced from Ref 41. 


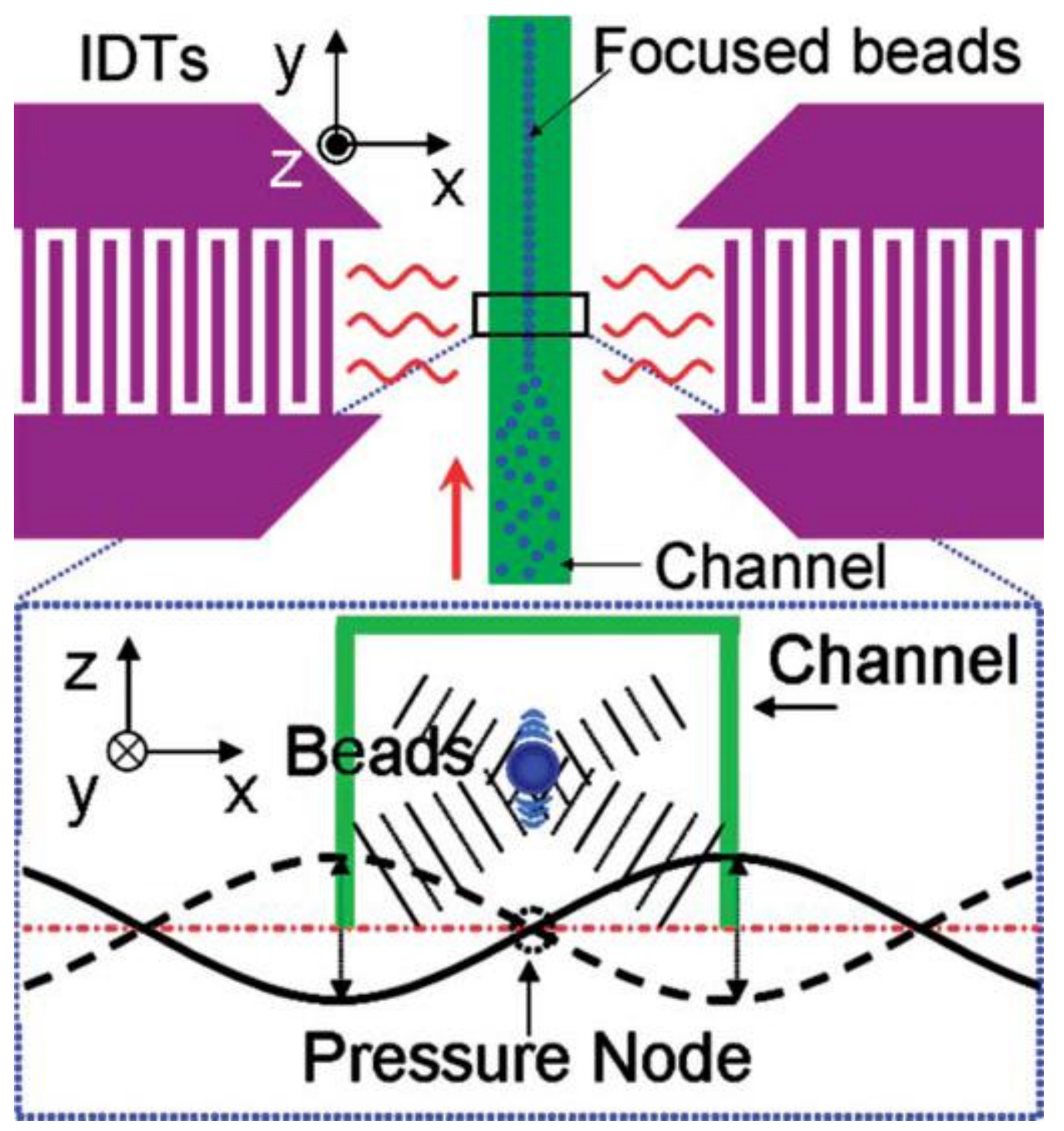

Figure 11. Schematic representation of the focusing device described by Shi et al. $^{44}$. Counter propagating surface acoustic waves are used to generate a point of low pressure in a PDMS fluid chamber. This forms a beam of particles along the length of the fluid channel. Higher frequencies will confine the particles more effectively but limits the channel size if a single beam of particles is required. Reproduced from Ref 44 . 